\title{
Analysis of stability robustness for generalized state-space systems with structured perturbations*
}

\section{Chun-Hsiung Fang}

Department of Electrical Engineering, National Kaohsiung Institute of Technology, 415 Chien-Kung Road, Kaohsiung 807. Taiwan, $R O C$

\section{Fan-Ren Chang}

Department of Electrica! Engineering, Nationul Taiwan University, Taipei 107, Taiwan, ROC

Received 15 August 1992

Revised 19 December 1992

\begin{abstract}
A new approach is proposed to analyze the stability robustness of generalized state-space systems with structured perturbations. The presented method is computationally simple to use and can easily be calculated by computer. As far as we are aware, this paper seems to be the first one to solve the robust stability problems for generalized state-space systems with structured uncertainties. The robust stability problem of generalized state-space systems is more complicated than that of regular state-space systems because it needs consideration of not only stability robustness but also system regularity and impulse elimination. The latter two ones need not be considered in regular state-space systems.
\end{abstract}

Keywords: Robust stability; generalized state-space systems; generalized eigenvalues; structured perturbations

\section{Notations and introduction}

$\begin{array}{ll}\rho(M) & : \text { spectral radius of } M \in C^{n \times n} \\ |M|_{\mathrm{m}} & : \text { modulus matrix of } M \in C^{n \times n} \\ |M| & : \text { determinant of matrix } M \in C^{n \times n} \\ \left|t_{i j}\right| & : \text { the }(i, j) \text { th element of } T \in \Re^{m \times n} \\ T \geq N & :\left[t_{i j}\right] \geq\left[n_{i j}\right] \text { for } i=1,2, \ldots, n, j=1, \\ & 2, \ldots, n \\ \operatorname{Re}(s) & : \text { real part of a complex number } s\end{array}$

Correspondence to: C.-H. Fang, Department of Electrical Engineering, National Kaohsiung Institute of Technology, 415 Chien-Kung Road, Kaohsiung 807, Taiwan, ROC.

* This work was supported by the National Science Council of Taiwan, ROC under Grant No. NSC-81-0404-E-151-513.
In recent years, there has been a growing interest in the system-theoretic problems of generalized state-space systems (or singular systems, or descriptor systems) due to the extensive applications of generalized state-space systems in large-scale systems, circuits, economics, control theory, and other areas $[5,9,10,17]$. Several important and fundamental results, except about robustness property, in regular systems have been successfully extended to generalized state-space systems $[1,6-8,12,18,20]$. In this paper we consider a continuous-time perturbed generalized state-space system described by

$$
E \dot{x}(t)=A x(t)+\Delta A x(t), \quad E x(0)=E x_{0},
$$

where $E, A \in \mathfrak{R}^{n \times n}$ and $\Delta A$ stands for the perturbations. Here the matrix $E$ may be singular. It usually represents system's structure. We will assume rank $E \equiv r \leq n$. Generally, system's perturbation can be characterized by using structured perturbations or unstructured perturbations $[2,3,13-16]$. Structured perturbations are those for which bounds on the individual elements of the perturbation matrix are known (or derived), whereas unstructured perturbations are those for which only a norm bound on the perturbation matrix is known (or derived). In system (1), $\Delta A$ denotes an $n \times n$ time-invariant structured perturbation matrix. The perturbations in the various elements of the system matrix are independent of one another. In a practical situation, the perturbation matrix is not known exactly and only the magnitude of the deviation that can be expected in the entries of $A$ may be known. Suppose the perturbation can be bounded by

$|\Delta A|_{\mathrm{m}} \leq k H$,

where $k$ is a real positive number and $H$ is a constant nonnegative matrix. The constant matrix $H$ represents the highly structured information for the additive perturbation matrix $\Delta A$. In this paper, the nominal system $(E, A)$ is assumed to be 
asymptotically stable and impulse-free, and the perturbation $\Delta E$ on matrix $E$ will not be considered. The cases that nominal singular system is not impulsive-free and $\Delta E$ exists are discussed in Remark 2.11.

If $E=I$ or $E$ is nonsingular, system (1) becomes the well-known perturbed regular state-space system. The robust control problems of regular statespace systems under structured uncertainties have been solved by many authors $[2,3,14-16,19]$. However, when $E$ is singular, the problems of stability robustness are still open and unsolved. The stability robusiness analysis for generalized statespace systems with structured uncertainties has not been dealt with. In this paper, we extend Chou's [3] results for the generalized system (1) with structured uncertainties. Since $E$ is not restricted to the identity matrix, the results of [3] may be viewed as a special case of ours.

It is well known that the system (1) contains three kinds of modes: finite dynamical modes, infinite dynamical modes, and infinite nondynamical modes $[1,17]$. The infinite dynamical modes can generate undesired impulse behaviors. Hence, to eliminate or to avoid inducing infinite dynamical modes is a key work in generalized state-space system control $[8,12,18]$. If we assume $\operatorname{deg}|s E-A|=r$ and $\Delta A=0$, system (1) now has $r$ finite dynamical modes, none of infinite dynamical modes, and $n-r$ infinite nondynamical modes. However, if the perturbation $\Delta A \neq 0$, it would possibly introduce dynamical infinite modes into system (1) since it can change the degree of $|s E-A-\Delta A|$. Furthermore, the perturbation $\Delta A$ can also possibly destroy the system's regularity (i.e. $|s E-A-\Delta A|$ is identically zero). For example, let

$$
\begin{aligned}
& E=\left[\begin{array}{ll}
1 & 1 \\
0 & 0
\end{array}\right], \quad A=\left[\begin{array}{cc}
-2 & -2 \\
4 & 4.2
\end{array}\right], \\
& \Delta A_{1}=\left[\begin{array}{cc}
0.1 & 0.1 \\
0.2 & 0
\end{array}\right], \quad \Delta A_{2}=\left[\begin{array}{cc}
0 & 0.1 \\
0 & -0.2
\end{array}\right] .
\end{aligned}
$$

Here $\operatorname{deg}|s E-A|=1=\operatorname{rank} E$. It is easy to check $\operatorname{deg}\left|s E-A-\Delta A_{2}\right|=0<\operatorname{rank} E$ (i.e. with infinite dynamical modes) and $\left|s E-A-\Delta A_{1}\right|$ is identically zero (i.e. without regularity). Hence, the robust stability analysis of uncertain generalized state-space systems should consider not only stability robustness but also system regularity and impulse-free behaviors. The latter two problems do not arise in regular state-space systems.

\section{Robust stability analysis}

Lemma 2.1. (Bender and Laub [1] and Verghese et al. [17]). The response of $E \dot{x}(t)=A x(t)$ is said to be asymptotically stable and impulse-free if and only if the following two conditions are satisfied:

$\operatorname{deg}|s E-A|=\operatorname{rank} E=r$

and

all roots of

$|s E-A|=0$

have negative real parts.

Equation (4) in Lemma 2.1 can be rewritten as

$|s E-A| \neq 0$ for all $\operatorname{Re}(s) \geq 0$.

Note that when $E=I$ or $E$ is nonsingular, equation (3) is always satisfied.

Definition 2.2. For any real constant matrices $X, Y \in \mathfrak{R}^{n \times n}$, the pair $(X, Y)$ is said to be asymptotically stable and impulse-free if $\operatorname{deg}|s X-Y|$ $=r$ and all roots of $|s X-Y|=0$ have negative real parts.

Lemma 2.3. (Ortega [11]). For any $n \times n$ constant complex matrices $X, Y, Z$ with $|X|_{m} \leq Z$, we have

$$
\begin{aligned}
& |X Y|_{\mathrm{m}} \leq|X|_{\mathrm{m}}|Y|_{\mathrm{m}} \leq Z|Y|_{\mathrm{m}}, \\
& |X+Y|_{\mathrm{m}} \leq|X|_{\mathrm{m}}+|Y|_{\mathrm{m}} \leq Z+|Y|_{\mathrm{m}}, \\
& \rho(X) \leq \rho\left(|X|_{\mathrm{m}}\right) \leq \rho(Z), \\
& \rho(X Y) \leq \rho\left(|X|_{\mathrm{m}}|Y|_{\mathrm{m}}\right) \leq \rho\left(Z|Y|_{\mathrm{m}}\right), \\
& \rho(X+Y) \leq \rho\left(|X+Y|_{\mathrm{m}}\right) \\
& \quad \leq \rho\left(|X|_{\mathrm{m}}+|Y|_{\mathrm{m}}\right) \leq \rho\left(Z+|Y|_{\mathrm{m}}\right) .
\end{aligned}
$$

For the perturbed system (1), assume that the pair $(E, A)$ is asymptotically stable and impulsefree, one can expand $(s E-A)^{-1}$ as

$(s E-A)^{-1}=G_{\mathrm{sp}}(s)+J$,

where $G_{\mathrm{sp}}(s)$ is a strictly proper rational matrix which is analytic in right-half $s$-plane and $J$ is a constant matrix. Denote by $G(t)$ the impulse response of $G_{\mathrm{sp}}(s)$ and define

$T \equiv \int_{0}^{\infty}|G(t)|_{\mathrm{m}} \mathrm{d} t$ 
The matrix $T$ would be finite since $G_{\mathrm{sp}}(s)$ is stable and strictly proper. A simple method to evaluate the matrix $T$ is given in Remark 2.5 .

Lemma 2.4. If the pair $(E, A)$ is asymptotically stable and impulse-free, then

$\left|(s E-A)^{-1}\right|_{m} \leq T+|J|_{m}$ for all $\operatorname{Re}(s) \geq 0$.

Proof. Taking Laplace transform of $G(t)$, we have

$G_{\mathrm{sp}}(s)=\int_{0}^{\infty} G(t) \mathrm{e}^{-s t} \mathrm{~d} t$

By Lemma 2.3 and equation (9), it is easy to check $\left|(s E-A)^{-1}\right|_{m} \leq\left|G_{s p}(s)\right|_{m}+|J|_{m}$

for all $\operatorname{Re}(s) \geq 0$

$\leq \int_{0}^{\infty}\left|G(t) \mathrm{e}^{-s t}\right|_{m} \mathrm{~d} t+|J|_{m}$ for all $\operatorname{Re}(s) \geq 0$

$\leq \int_{0}^{\infty}|G(t)|_{m} d t+|J|_{m}$ for all $\operatorname{Re}(s) \geq 0$

$=T+|J|_{m}$.

Remark 2.5. We propose a simple method to evaluate $G(t)$ by the Weierstrass decomposition $[5,17]$. Assume that the pair $(E, A)$ is asymptotically stable and impulse-free. It can be transformed to the well-known Weierstrass form, i.e. there exist two constant nonsingular matrices $U$ and $V$ such that

$U(s E-A) V=\left[\begin{array}{cc}s I_{r}-A_{r} & 0 \\ 0 & I_{n-r}\end{array}\right]$,

where $A_{r} \in \Re^{r \times r}$. Suppose $U$ and $V$ are decomposed as

$U=\left[\begin{array}{l}U_{a} \\ U_{b}\end{array}\right], \quad V=\left[\begin{array}{ll}V_{a} & V_{b}\end{array}\right]$,

where $U_{a} \in \Re^{r \times n}, \quad U_{b} \in \Re^{(n-r) \times r}, \quad V_{a} \in \Re^{n \times r}$, and $V_{b} \in \Re^{n \times(n-r)}$. Then it is easy to show that

$$
\begin{aligned}
G_{s p}(s) & =V_{a}\left(s I_{r}-A_{r}\right)^{-1} U_{a}, \\
J & =V_{b} \cdot U_{b} .
\end{aligned}
$$

From equation (16a), we have

$$
G_{\mathrm{sp}}(s)=V_{a}\left[\int_{0}^{\infty} \mathrm{e}^{A_{r} t} \mathrm{e}^{-s t} \mathrm{~d} t\right] U_{a} .
$$

A comparison with (12) gives

$G(t)=V_{a} \mathrm{e}^{A_{r} t} U_{a}$.

Lemma 2.6. For a matrix $Q \in C^{n \times n}$, if $\rho(Q)<1$, then $|I \mp Q| \neq 0$.

Theorem 2.7. Assume the perturbation $\Delta A$ can be bounded by $|\Delta A|_{m} \leq k H$. The perturbed system (1) is asymptotically stable and impulse-free if the pair $(E, A)$ is asymptotically stable and impulse-free, and the following inequality is satisfied:

$k<\frac{1}{\rho\left(T H+|J|_{\mathrm{m}} H\right)}$.

Proof. By the determinant formula, we have

$$
\begin{aligned}
|s E-A-\Delta A|= & |s E-A| \cdot \mid I \\
& -(s E-A)^{-1} \Delta A \mid .
\end{aligned}
$$

From Lemma 2.3 and equation (13), the following inequalities are obvious,

$$
\begin{aligned}
& \rho\left((s E-A)^{-1} \Delta A\right) \\
& \quad \leq \rho\left(\left|(s E-A)^{-1}\right|_{\mathrm{m}} k H\right) \quad \text { for all } \operatorname{Re}(s) \geq 0 \\
& \quad \leq \rho\left(k|J|_{\mathrm{m}} H+k T H\right) \text { for all } \operatorname{Re}(s) \geq 0 .
\end{aligned}
$$

If equation (19) is satisfied, then $\rho((s E-$ $\left.A)^{-1} \Delta A\right)<1$ for all $\operatorname{Re}(s) \geq 0$. According to Lemma 2.6, we have

$\left|I-(s E-A)^{-1} \Delta A\right| \neq 0$ for all $\operatorname{Re}(s) \geq 0$.

Hence, we obtain $|s E-A-\Delta A| \neq 0$ for all $\operatorname{Re}(s) \geq 0$ from equation (20). Using equations (9) and (20), it is easy to verify that

$$
\begin{aligned}
& |s E-A-\Delta A| \\
& \quad=|s E-A|\left|I-J \Delta A-G_{\mathrm{sp}}(s) \Delta A\right| .
\end{aligned}
$$

Since $G_{\mathrm{sp}}(s)$ is a stable and strictly proper rational matrix, the constant term of $\left|I-J \Delta A-G_{\mathrm{sp}}(s) \Delta A\right|$ will be decided by $(I-J \Delta A)$. If $(I-J \Delta A)$ is nonsingular, we can obtain, from equation (23),

$$
\operatorname{deg}|s E-A-\Delta A|=\operatorname{deg}(|s E-A||I-J \Delta A|) \text {. }
$$

By Lemma 2.3, it is easy to show

$$
\rho(J \Delta A) \leq \rho\left(k|J|_{\mathrm{m}} H\right) \leq \rho\left(k T H+k|J|_{\mathrm{m}} H\right) .
$$

If inequality (19) is satisfied, $\rho(J \Delta A)<1$, and $(I-J \Delta A)$ is nonsingular. Then from equation (24), 
we have $\operatorname{deg}|s E-A-\Delta A|=\operatorname{deg}|s E-A|=r$. Therefore, the perturbed system (1) is asymptotically stable and impulse-free. Furthermore, if inequality (19) holds, equations (20) and (24) can guarantee the regularity of the perturbed system.

Remark 2.8. From the above statements, we know that the regularity can be guaranteed if the perturbed generalized state-space system is asymptotically stable and impulse-free.

Remark 2.9. If $E=I$, then $J=0$. The sufficient condition in equation (19) coincides with the results in [3]. Thus, Chou's results might be viewed as a special case of ours.

Remark 2.10. One can use the criterion developed in this paper to design a robust controller for an uncertain generalized state-space system. Assume that the given perturbed system is $E \dot{x}(t)=$ $A x(t)+\Delta A x(t)+B u(t)$, where the triple $(E, A, B)$ is strongly controllable [17]. We can apply a state feedback $u(t)=F x(t)$ such that the pair $(E, A+B F)$ is asymptotically stable and impulsefree $[8,18]$. Then one may use the inequality $(19)$ to check whether the designed feedback gain $F$ is robust under the perturbation $\Delta A$.

Remark 2.11. If the nominal system is not impulsefree, it can be theoretically decomposed by nonsingular matrtix transformation as

$U(s E-A) V=\left[\begin{array}{cc}s I_{p}-A_{p} & 0 \\ 0 & s J+I_{n-p}\end{array}\right]$,

where $p<\operatorname{rank} E, A_{p} \in R^{p \times p}, J$ is a nilpotent Jordan matrix, and both $U$ and $V$ are nonsingular constant matrices. In this case, an "arbitrarily small" matrix $A_{s}$ of the form

$U A_{s} V=\left[\begin{array}{cc}0 & 0 \\ 0 & A_{s 4}\end{array}\right]$

can be constructed such that $\operatorname{deg} \mid s J+I_{n-p}+$ $A_{s 4} \mid=\operatorname{rank}(J)$ (i.e. $\operatorname{deg}\left|s E-A+A_{s}\right|=\operatorname{rank} E$ ) and all roots of $\left|s J+I_{n-p}+A_{s 4}\right|=0$ lie in the left-half plane. By the $A_{s}$, we can rewrite the perturbed singular system as

$$
\begin{aligned}
E \dot{x}(t) & =A x(t)+\Delta A x(t) \\
& =\left(A-A_{s}\right) x(t)+\left(A_{s}+\Delta A\right) x(t) \\
& =\hat{A} x(t)+\Delta \hat{A} x(t),
\end{aligned}
$$

where $\hat{A} \equiv A-A_{s}$ and $\Delta \hat{A} \equiv \Delta A+A_{s}$. Now the new nominal system $(E, \hat{A})$ is asymptotically stable and impulse-free and the $\Delta \hat{A}$ may be viewed as a new structured perturbation on matrix $\hat{A}$. Note that here $A_{s}$ is a "selected arbitrarily small" matrix.

In many applications, the matrix $E$ is a structure information matrix rather than a parameter matrix, i.e. the elements of $E$ contain only structure information regarding the problem considered. Hence, they are not subject to variations. In the case when the matrix $E$ does contain uncertain parameters and when the perturbation is of the form

$U(\Delta E) V=\left[\begin{array}{cc}\Delta E_{1} & \Delta E_{2} \\ 0 & \Delta E_{4}\end{array}\right]$,

then we have, from equation (14),

$$
\begin{aligned}
|s(E+\Delta E)-A|= & \left|U^{-1}\right| \cdot\left|V^{-1}\right| \cdot \mid s\left(I_{r}+\Delta E_{1}\right) \\
& -A_{r}|\cdot| s \Delta E_{4}+I_{n-r} \mid \cdot
\end{aligned}
$$

In equation (30) we assume that the nominal system is stable and impulse-free. If $\left|s\left(I_{r}+\Delta E_{1}\right)-A_{r}\right|$ is not identically zero, one can find an "arbitrarily small" matrix $\Delta E_{4}$ such that $\left|s \Delta E_{4}+I_{n-r}\right|=0$ has at least one root in the right-half plane. Hence, in this case the stability of $(E, A)$ has zero tolerance to $\Delta E_{4}$ on matrix $E$. The influence of $\Delta E_{1}$ to system stability can be checked by using

$\left|s\left(I_{r}+\Delta E_{1}\right)-A_{r}\right|=s^{r}\left|\left(I_{r}+\Delta E_{1}\right)-\lambda A_{r}\right|$,

where $s=1 / \lambda$, and $A_{r}$ is a stable matrix. $A_{r}$ is nonsingular implies that $\left|\left(I_{r}+\Delta E_{1}\right)-\lambda A_{r}\right|=0$ always has $r$ finite roots for arbitrary $\Delta E_{1}$. If all $r$ roots of $\left|\left(I_{r}+\Delta E_{1}\right)-\lambda A_{r}\right|=0$ have negative real parts for perturbation $\Delta E_{1}$, we may say that all $r$ roots of $\left|s\left(I_{r}+\Delta E_{1}\right)-A_{r}\right|=0$ also have negative real parts for perturbation $\Delta E_{1}$. If we define $\tilde{E} \equiv A_{r}, \tilde{A} \equiv I_{r}$, and $\Delta \tilde{A} \equiv \Delta E_{1}$, we may use the method proposed in Theorem 2.7 to check whether all $r$ roots of $\left|I_{r}+\Delta E_{1}-\lambda A_{r}\right|=0$ lie in left-half plane.

Recently, Qiu and Davison [13] have dealt with problem same as those considered in this paper. However, the perturbation $\Delta A$ on matrix $A$ they investigated is unstructured (measured by spectral norm). They also assumed that the perturbation $\Delta E$ on matrix $E$ is zero and the nominal system $(E, A)$ is asymptotically stable and impulse-free. We would like to refer to [13] for a discussion of the assumptions made by these authors. 


\section{Illustrative examples}

Example 3.1. Let the nominal system be

$E=\left[\begin{array}{lll}1 & 0 & 0 \\ 0 & 1 & 0 \\ 0 & 0 & 0\end{array}\right], \quad A=-\left[\begin{array}{lll}1 & 0 & 0 \\ 0 & \varepsilon & 0 \\ 0 & 0 & \varepsilon\end{array}\right]$,

where $\varepsilon$ is a real positive number. Assume that the perturbation is bounded by

$|\Delta A|_{\mathrm{m}} \leq \varepsilon \cdot\left[\begin{array}{lll}0 & 0 & 0 \\ 0 & 1 & 1 \\ 0 & 1 & 1\end{array}\right]=k \cdot H$

where $k=\varepsilon$. It is easy to get

$T=\left[\begin{array}{lll}1 & 0 & 0 \\ 0 & \frac{1}{\varepsilon} & 0 \\ 0 & 0 & 0\end{array}\right], \quad J=\left[\begin{array}{lll}0 & 0 & 0 \\ 0 & 0 & 0 \\ 0 & 0 & \frac{1}{\varepsilon}\end{array}\right]$.

We then have $\rho\left(T H+|J|_{m} H\right)=2 / \varepsilon$. Since

$k=\varepsilon \nless \frac{1}{\rho\left(T H+|J|_{\mathrm{m}} H\right)}=\frac{\varepsilon}{2}$,

we cannot make any conclusion for this perturbation according to the sufficient condition proposed in Theorem 2.7. In fact, if we let $\Delta A=\varepsilon \cdot H$, it is easy to check $\operatorname{deg}|s E-A|=2>\operatorname{deg} \mid s E-A$ $-\Delta A \mid=1$. Obviously, this perturbation introduces a dynamical impulsive mode to the nominal system.

Example 3.2. Consider the following system:

$$
\begin{aligned}
& {\left[\begin{array}{lll}
1 & 0 & 0 \\
0 & 0 & 0 \\
0 & 1 & 0
\end{array}\right] \dot{x}(t) } \\
= & {\left[\begin{array}{ccc}
-8 & 0 & 0 \\
4 & 0 & -2 \\
0 & -1 & 0
\end{array}\right] x(t)+\Delta A x(t), }
\end{aligned}
$$

where the pair $(E, A)$ is asymptotically stable and impulse-free. By Weierstrass decomposition, it is easy to obtain

$U=\left[\begin{array}{ccc}1 & 0 & 0 \\ 0 & 0 & 1 \\ 0 & 0.5 & 0\end{array}\right], \quad V=\left[\begin{array}{lll}1 & 0 & 0 \\ 0 & 1 & 0 \\ 2 & 0 & 1\end{array}\right]$ then we have

$A_{r}=\left[\begin{array}{cc}-8 & 0 \\ 0 & -1\end{array}\right], \quad|J|_{\mathrm{m}}=\left[\begin{array}{ccc}0 & 0 & 0 \\ 0 & 0 & 0 \\ 0 & 0.5 & 0\end{array}\right]$

and

$G(t)=\left[\begin{array}{ccc}\mathrm{e}^{-8 t} & 0 & 0 \\ 0 & 0 & \mathrm{e}^{-t} \\ 2 \mathrm{e}^{-8 t} & 0 & 0\end{array}\right], \quad T=\left[\begin{array}{ccc}\frac{1}{8} & 0 & 0 \\ 0 & 0 & 1 \\ \frac{1}{4} & 0 & 0\end{array}\right]$.

Given the structured perturbation information the allowable upper bounds on $k$ are as follows:

if $H=\left[\begin{array}{lll}1 & 0 & 0 \\ 0 & 0 & 0 \\ 0 & 0 & 0\end{array}\right], \quad \frac{1}{\rho\left(T H+|J|_{\mathrm{m}} H\right)}=8$

(i.e. the perturbed generalized state-space system in this example is asymptotically stable and impulse-free if the perturbation is bounded by $|\Delta A|_{\mathrm{m}} \leq 8 \cdot H$. In fact, the constant 8 is the exact upper bound for the structured perturbation).

if $H=\left[\begin{array}{lll}0 & 0 & 0 \\ 0 & 0 & 1 \\ 0 & 0 & 0\end{array}\right], \quad \frac{1}{\rho\left(T H+|J|_{\mathrm{m}} H\right)}=2$.

The constant 2 is the exact upper bound for the structured perturbation. Another structured perturbations bounds are calculated in the following:

$$
\begin{aligned}
\text { if } H=\left[\begin{array}{lll}
0 & 0 & 0 \\
0 & 0 & 0 \\
0 & 0 & 1
\end{array}\right], \frac{1}{\rho\left(T H+|J|_{\mathrm{m}} H\right)}=\infty, \\
\text { if } H=\left[\begin{array}{lll}
H & 0 & 0 \\
0 & 1 & 0 \\
0 & 0 & 1
\end{array}\right], \frac{1}{\rho\left(T H+|J|_{\mathrm{m}} H\right)}=1.414,
\end{aligned}
$$$$
\text { if } H=\left[\begin{array}{lll}
0 & 0 & 1 \\
0 & 1 & 0 \\
1 & 0 & 0
\end{array}\right], \frac{1}{\rho\left(T H+|J|_{\mathrm{m}} H\right)}=2 \text {, }
$$

if $H=\left[\begin{array}{lll}1 & 0 & 1 \\ 0 & 0 & 0 \\ 1 & 0 & 1\end{array}\right], \quad \frac{1}{\rho\left(T H+|J|_{\mathrm{m}} H\right)}=2.6667$, 
if $H=\left[\begin{array}{lll}0 & 1 & 0 \\ 1 & 0 & 1 \\ 0 & 1 & 0\end{array}\right], \frac{1}{\rho\left(T H+|J|_{\mathrm{m}} H\right)}=1$ and
if $H=\left[\begin{array}{lll}1 & 1 & 1 \\ 1 & 1 & 1 \\ 1 & 1 & 1\end{array}\right], \frac{1}{\rho\left(T H+|J|_{\mathrm{m}} H\right)}=0.533$.

\section{Conclusions}

A new and simple approach is proposed to analyze the stability robustness for generalized statespace systems with structured perturbations. The robust stability problem of generalized state-space system is more complicated than that of regular state-space system because not only stability robustness but also regularity preservation and impulse elimination should be simultaneously considered. Our method only requires evaluation of a time-invariant stable transition matrix. So it is computationally simple and can easily be calculated by a computer. To the best of authors' knowledge, the paper seems to be the first one to solve the stability robustness problem for generalized state-space system with structured perturbations. Research is ongoing in order to extend the proposed robustness criteria to the design problem of feedback generalized state-space systems. Extension of the results in this paper to nonsquare generalized state-space systems is a topic for future research.

\section{Acknowledgment}

The authors gratefully acknowledge the reviewers for their suggestions and comments.

\section{References}

[1] D.J. Bender and A.J. Laub, The linear-quadratic optimal regulator for descriptor systems, IEEE Trans. Automat. Control 32 (1981) 672-687.
[2] B.S. Chen and C.C. Wong, Robust linear controller design: time domain approach, IEEE Trans. Automat. Control 32 (1987) 161-164.

[3] J.H. Chou, Stability robustness of linear state space models with structured perturbations, Systems Control Lett. 15 (1990) 207-210.

[4] J.D. Cobb, Controllability observability, and duality in singular systems, IEEE Trans. Automat. Control 29 (1984) 1076-1082.

[5] L. Dai, Singular Control Systems, Lecture Notes in Control and Information Sciences (Springer, Berlin, 1989).

[6] C.H. Fang and F.R. Chang, Realization algorithm for constructing a controllable representation of a singular system with a special coordinate, Internat. J. Control 50 (1989) 1217-1226.

[7] C.H. Fang and F.R. Chang, Deadbeat control in singular systems and its applications, Control Theory Adv. Tech. 6 (1990) 383-393.

[8] V. Kucera and P. Zagalak, Fundamental theorems of state feedback for singular systems, Automatica 24 (1988) 653-658.

[9] F.L. Lewis, A survey of linear singular systems, J. Circuit Syst. Signal Process. 5 (1986) 3-36.

[10] D.G. Luenberger, Dynamic equations in descriptor form, IEEE Trans. Automat. Control 22 (1977) 312-321.

[11] J.M. Ortega, Numerical Analysis (Academic Press, New York, 1972).

[12] P.N. Paraskevopoulos and F.N. Koumboulis, Decoupling and pole assignment in generalized state space systems, IEE Pt.D 138 (1991) 547-560.

[13] L. Qiu and E.J. Davison, The stability robustness of generalized eigenvalues, IEEE Trans. Automat. Control 37 (1992) 886-891.

[14] A. Rachid, Robustness of discrete systems under structured uncertainties, Internat. J. Control 50 (1989) 1563-1566.

[15] C.B. Soh, Stability robustness measures of state-space models, Internat. J. System Sci. 22 (1991) 1867-1884.

[16] K.M. Sobel, S.S. Banda and H.H. Yeh, Robust control for linear systems with structured state space uncertainty, Internat. J. Control 50 (1989) 1991-2004.

[17] G.C. Verghese, B.C. Levy and T. Kailath, A generalized state-space for singular systems, IEEE Trans Automat. Control 26 (1981) 811-831.

[18] Y.Y. Wang, S.J. Shi and Z.J. Zhang, Pole placement and compensator design of generalized systems, Systems Control Lett. 8 (1987) 205-209.

[19] R.K. Yedavalli, Improved measures of stability robustness for linear state space models, IEEE Trans. Automat. Control 30 (1985) 577-579.

[20] Z. Zhou, M.A. Shayman and T.J. Tarn, Singular systems: a new approach in the time domain, IEEE Trans. Automat. Control 32 (1987) 42-50. 